\title{
BUNDLE ADJUSTMENT OF SPACEBORNE DOUBLE-CAMERA PUSH-BROOM IMAGERS AND ITS APPLICATION TO LROC NAC IMAGERY
}

\author{
I. Haase ${ }^{1, *}$, P. Gläser ${ }^{1,2}$, J. Oberst ${ }^{1,3}$ \\ ${ }^{1}$ Technical University Berlin (TUB), Dept. of Planetary Geodesy, 10623 Berlin, Germany - (isabel.haase, philipp.glaeser, \\ juergen.oberst)@tu-berlin.de \\ ${ }^{2}$ Ronin Institute for Independent Scholarship, Montclair, NJ 07043, USA \\ ${ }^{3}$ German Aerospace Center (DLR), Institute of Planetary Research, 12489 Berlin, Germany
}

Commission III, ICWG III/II

KEY WORDS: Bundle Adjustment, DTM, orthomosaic, LROC, LOLA, co-registration, variance component estimation, Moon

\begin{abstract}
:
The TU Berlin group of the Lunar Reconnaissance Orbiter Camera (LROC) team has implemented a Bundle Adjustment (BA) for spaceborne multi-lenses line scan imagers, by rigorously modeling the geometric properties of the image acquisition. The BA was applied to stereo image sets of the LROC Narrow Angle Camera (NAC) and first results show, that the overall geometry of the stereo models were significantly improved. Ray intersection accuracies of initially up to several meters were homogenized within the integrated stereo models and improved to $0.14 \mathrm{~m}$ on average. The mean point error of the adjusted 3D object points was estimated by the BA to be $0.95 \mathrm{~m}$. The inclusion of available Lunar Orbiter Laser Altimeter (LOLA) shots as 3D ground control to the BA, accurately tied to the image space by an aforegoing co-registration, allowed to register the final adjusted NAC DTM to the currently most accurate global lunar reference frame. The BA also provides accuracy assessments of the individual LOLA tracks used for georeference during the adjustment, which will be useful to further assess LOLA derived products.
\end{abstract}

\section{INTRODUCTION}

Planetary image data provided by camera systems flown on spacecrafts are used to map the surfaces of distant planetary bodies and to derive digital terrain models (DTMs) and orthoimage maps. High-quality and high-resolution topographic and cartographic products are of fundamental importance in planetary science as spatial data evaluations and comparisons rely on accurate geo-referencing, and they are evidently critical to accurate and safe landings (or rendezvous maneuvers).

In order to derive topographic information from planetary images, photogrammetric techniques are applied, which involve several successive steps:

- $\quad$ radiometric calibration of the images,

- determination of a dense network of tie-points by automatic image-matching,

- 3D object point determination by forward ray intersections, and finally

- DTM interpolation.

The fundamental requirement for the exploitation of 3D information from images is the exact knowledge of the camera geometry (interior orientation) and the position and orientation of the camera during image acquisition (exterior orientation). In extraterrestrial photogrammetry, these values are usually provided by a camera calibration under laboratory conditions prior to launch and by Navigation and Ancillary Information Facility (NAIF) SPICE kernels providing the spacecraft ephemerides and camera attitudes as functions of time. If these parameters are not known with sufficient accuracy, the derived products are systematically corrupted and can only be regarded as a first approximation to reality.

A way to improve the a priori known parameters is a BA that rigorously models the geometric recording situation as realistically as possible and provides improved values for the interior and exterior orientation parameters (as well as adjusted
3D object points). Here we present the BA approach developed at the TU Berlin, specifically designed for extraterrestrial images provided by spaceborne multi-lenses push-broom scanners such as the double-camera system LROC NAC (Robinson et al., 2010) of the Lunar Reconnaissance Orbiter (LRO) mission (Keller et al., 2016).

\section{CHALLENGES IN EXTRATERRESTRIAL PHOTOGRAMMETRY}

Contrary to handling conventional images provided by frame cameras, the implementation of a BA for spaceborne line-scan images is challenging and not always unique in its solution. There are different approaches, and depending on how accurately and precisely the reality and the different sources of errors are modeled (the functional and stochastic model), a BA will provide more or less accurate estimates of the ground truth.

In general, statements about the precision achieved by an adjustment are only meaningful, if the functional model is complete, the observations are free of gross errors, and the stochastic model is correct. From our experience, the main challenges in evaluating planetary images are the appropriate modeling of the trajectory of the orbiter and its attitude, the inclusion of geodetic (ground) control information of superior accuracy (typically missing on extraterrestrial bodies), and the a priori estimation of the accuracy of the observations (the stochastic model).

\section{LROC NAC}

LROC NAC is a camera system of two Charge-Coupled Device (CCD) linear arrays, which image the lunar surface line by line as LRO moves forward on its polar lunar orbit. Each sensor has its own optics and consists of 5,064 pixels that are $7 \mu \mathrm{m}$ wide. With an orbit altitude of $50 \mathrm{~km}$, which used to be the mean altitude during the primary mission, the ground sampling distance of a typical NAC image is $0.5 \mathrm{~m}$. LROC is not a stereo

\footnotetext{
* Corresponding author
} 
camera in the conventional sense, as the two cameras (NACLeft and NAC-Right) do not provide a forward and backward view nor use scanning mirrors or any other moving parts. The two cameras cover areas adjacent to each other with a small overlap in across-track direction. The small overlapping area of the two NACs of $\sim 135$ pixels does not provide appropriate stereo information because the perspective is nearly identical. However, this narrow "seam" is useful for the BA, since, together with a stereo partner from a different orbit, redundant observations are available, which geometrically stabilize the overall model.

The acquisition of a stereo image partner is performed by slewing the entire spacecraft in across-track direction and imaging the particular area of interest from a subsequent orbit, typically from the following one $(\Delta \mathrm{t} \approx 2 \mathrm{~h})$ or the one after $(\Delta \mathrm{t} \approx 4 \mathrm{~h})$. The resulting convergence angles range from $12^{\circ}$ to about $40^{\circ}$. A typical LROC NAC stereo image set consists of four images, two images per orbit. Depending on the geometry of image acquisition and the local topography, this configuration results in a maximum of four stereo models, which are referred to as $\mathrm{L}-\mathrm{L}, \mathrm{R}-\mathrm{R}, \mathrm{L}-\mathrm{R}$, and $\mathrm{R}-\mathrm{L}$ in the following. The resulting four object models are finally combined to a DTM.

\section{THE TUB BUNDLE ADJUSTMENT}

Our BA approach is based on a weighted least-squares estimation in the Gauss-Markov model. Its basic features are as follows and will be explained in more detail in the following sections:

- Gauss-Markov model

- $\quad$ extended collinearity equations

- $\quad 3^{\text {rd }}$ degree Lagrange Polynomials

- co-registered LOLA ground control

- $\quad$ outlier detection (Baarda’s Data Snooping)

- variance component estimation

\subsection{Initial DTM}

To derive 3D topography from LROC NAC images we use a modified version of the DLR stereo processing chain (Scholten et al., 2005), which was originally developed to process images acquired by the High Resolution Stereo Camera (HRSC) on board of the Mars Express orbiter. A detailed description of the generation of LROC NAC DTMs at TU Berlin is given by Haase et al. (2019).

An initial NAC DTM is processed for two reasons: First, it is used to generate orthoimages, which serve as input images for the DLR image matcher to retrieve tie points. In addition, the object point cloud derived from forward ray intersection is used to tie LOLA 3D points to the image space, which will be explained in more detail in paragraph 4.5. The exterior orientation used to process the initial DTM is based on the most recent NAIF SPICE kernels provided by the LRO Science Operation Center (SOC) and the instrument teams. Figure 1 shows the initial LROC NAC DTM of our study.

Experience shows that nominal SPICE spacecraft trajectory and pointing data suffer from residual errors, which result in DTMs with insufficient internal accuracy, which may lead to a slightly twisted and rotated surface model, point displacements, and noise. An important measure of the (relative) accuracy of the overall geometry of a stereo model is the mean intersection distance of the (forward) ray intersections. The intersection accuracies of the four NAC stereo models, i.e. L-L, R-R, L-R, and R-L, of the initial DTM presented here are very inhomogeneous, ranging from $1.3 \mathrm{~m}$ to $9.4 \mathrm{~m}$ (Table 1 , Figure 2a). They will be higly improved by our BA, as can be seen in Figure $2 \mathrm{~b}$ and Table 1, and as will be shown later.

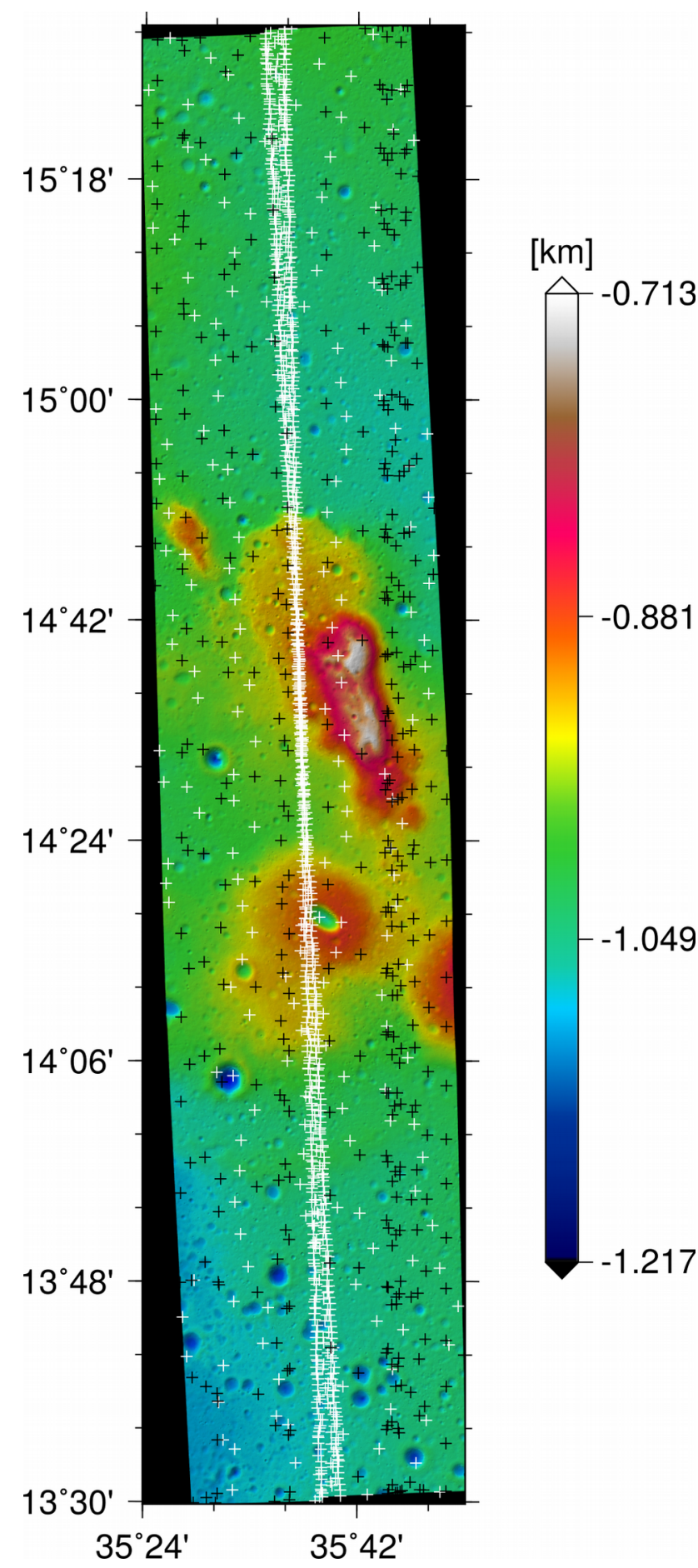

Figure 1: Initial $3 \mathrm{~m}$ DTM (shaded and color-coded) showing the locations of the LOLA ground control points (black plus signs) and the image tie points (white plus signs) used in the BA. Elevations refer to the mean lunar radius of $1737.4 \mathrm{~km}$.

Therefore, the insertion of a BA in the photogrammetric processing chain to improve the parameters of the exterior orientations is generally advisable, but is urgently necessary if the derived cartographic products have to meet the highest 
accuracy requirements, e.g. in the planning and execution of a precise and safe landing or as data basis of optical navigation systems.

\subsection{Extended Collinearity Equations}

The functional relationship between the image and the object space is described by the collinearity equations. We used the extended version of the collinearity functions as proposed by Ebner et al. (1992), to rigorously describe the relative alignment of the two cameras mounted on the same platform. We defined the NAC-L as the master sensor, for which coordinate transformations are directly performed between the image and the object space (Mean Earth/Polar Axis-, ME-frame) using the position of the NAC-L projection center $\left(\mathrm{X}_{0_{-} \mathrm{L}}, \mathrm{Y}_{0_{-} \mathrm{L}}, \mathrm{Z}_{0_{-} \mathrm{L}}\right)$ and the NAC-L rotation angles $\left(\omega_{\mathrm{L}}, \varphi_{\mathrm{L}}, \mathrm{K}_{\mathrm{L}}\right)$. For the NAC-R, on the other hand, we introduced an additional 6-parameter transformation to perform coordinate transformations between the NAC-R and the NAC-L. Coordinate transformations between the NAC-R and the object space therefore always involve the position and rotation of the NAC-L (master sensor) as well.

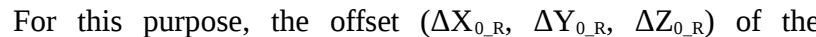
projection center of the NAC-R from the NAC-L projection center is determined with respect to the NAC-L coordinate frame. Furthermore, the rotations to transform image coordinates acquired in the NAC-R coordinate frame to the NAC-L frame and their according Euler angles $\Delta \omega_{R}, \Delta \varphi_{R}, \Delta \kappa_{R}$ are determined, one rotation per orbit. Practically, for all tie points that were measured in NAC-R images the extended collinearity equations are used (see Ebner et al., 1992, for further detail). For the master sensor (the NAC-L per our definition) the extended version is identical to the classical collinearity equations as the parameters of the NAC-R are zero and cancel out.

Offset values in the spacecraft bus frame for both LROC NAC cameras were measured by the instrument integration team after the cameras had been installed on the spacecraft. They are given in the frame kernel (fk). The pointing of the two NACs with respect to the spacecraft bus were also measured, but could be refined with in-flight calibration measurements and are provided by addendum C-kernels (ck) for each image by the LROC SOC (Speyerer et al., 2014).

In the BA, the parameters of the exterior orientations of both cameras are treated as unknowns and are additionally introduced as fictitious observations, allowing to associate weights to them and receiving an accuracy estimate after the computation. Usually, we introduce a very small standard deviation, i.e. a large weight, for the NAC-R offset, to more or less hold it fixed. All other exterior orientation parameters are allowed to improve, i.e. they are introduced to the BA with standard deviations determined by a pre-adjustment.

\subsection{Lagrange Polynomials}

To apply photogrammetric methods to images, exact knowledge of the parameters of the exterior orientation of the camera $\left(\mathrm{X}_{0}\right.$, $\left.\mathrm{Y}_{0}, \mathrm{Z}_{0}, \omega, \varphi, \kappa\right)$ is the fundamental prerequisite. In contrast to frame cameras, which have only one instance of image acquisition and therefore only one exterior orientation, the push-broom principle of linear array sensors results in one exterior orientation per line, 52,224 in the case of the LROC NAC. It would be impracticable, if not impossible, in terms of time and memory, to integrate all lines into the BA. Therefore, the six parameters of the exterior orientation are usually approximated by polynomials, one per parameter.

\begin{tabular}{|c|r|c|c|c|c|}
\hline NAC & \# of tie points & \multicolumn{2}{|c|}{ before adjustment } & \multicolumn{2}{|c|}{ after adjustment } \\
\hline & & $\begin{array}{c}\text { mean } \\
\text { inter- } \\
\text { section } \\
{[\mathrm{m}]}\end{array}$ & $\sigma[\mathrm{m}]$ & $\begin{array}{c}\text { mean } \\
\text { inter- } \\
\text { section } \\
{[\mathrm{m}]}\end{array}$ & $\sigma[\mathrm{m}]$ \\
\hline L-L & $238,385,258$ & 9.4 & 0.4 & 0.14 & 0.10 \\
\hline R-R & $238,026,663$ & 4.6 & 0.3 & 0.13 & 0.10 \\
\hline L-R & $12,256,020$ & 1.3 & 0.2 & 0.24 & 0.14 \\
\hline R-L & $10,948,016$ & 6.0 & 0.2 & 0.22 & 0.15 \\
\hline
\end{tabular}

Table 1. Mean ray intersections and their standard deviations before and after the bundle adjustment.

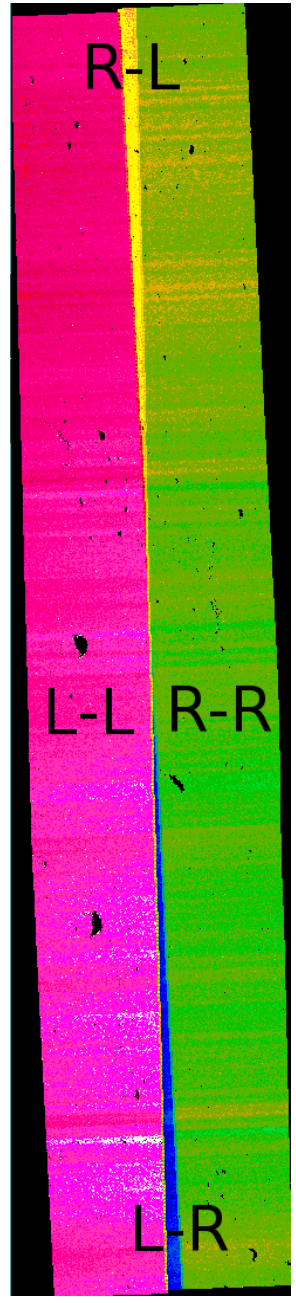

(a)

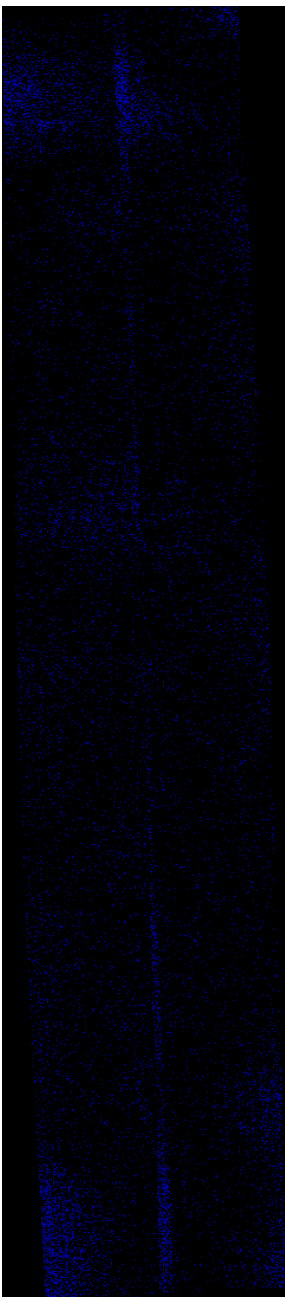

$10 \mathrm{~m}$

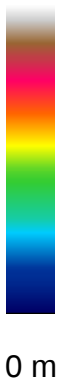

(b)
Figure 2: Color-coded representation of the intersection errors. (a) Initial errors of the camera positions and pointings result in high intersection errors of up to $\sim 10 \mathrm{~m}$. (b) After bundle adjustment the improved exterior orientations lead to significantly reduced intersection errors of $0.14 \mathrm{~m}$ on average.

To model the trajectory and attitude of LRO (or the master NAC-L camera, to be more precise) we select four image lines (here synonymously used for the epochs et $t_{i}$ ) which are equally distributed over the image. They serve as nodes of the cubic Lagrange polynomials to interpolate the exterior orientation parameters for all image lines. 
The advantage of using Lagrange interpolation polynomials is that the exterior orientation parameters can be introduced directly as unknowns to the adjustment. As with all polynomials, there is the risk of oscillation (Runge's phenomenon) at the interval edges if the degree of the polynomial (= number of nodes -1$)$ is chosen too high. Therefore, it is usually not advisable to increase the degree of the polynomial to more than 3 if the spacing between the nodes is to be reduced (e.g. by increasing the number of nodes). Here, an alternative solution may be to (piecewise) connect cubic polynomials, which we are currently testing.

\subsection{Interior Orientation}

Errors in the sensor model turn into systematic errors. Therefore, an accurate calibration must be carried out before launch to obtain the best possible a priori values for the principal point coordinates, the effective focal length, and possible optical (lens) distortion (interior orientation). The LROC team determined the boresight pixel, distortion coefficient, and focal length for each NAC during pre-flight calibration measurements (Robinson et al., 2010). However, due to strong physical forces during launch or the extreme environmental conditions in space, e.g. temperature changes, the calibrated values are usually not stable and can slightly change during the mission.

In theory, it is possible to simultaneously estimate the parameters of the interior orientation in the BA as part of a socalled on-orbit "self-calibration". In the LROC NAC case, due to the lack of high-precision control points and the unfavourable geometric arrangement of the images, which result in high correlations between the parameters of the interior orientation, we have not pursued this approach any further (practically, our BA is capable of doing so). Therefore, our results may be affected by errors in the interior orientation, which should be considered when interpreting the results and looking for possible further improvement.

\subsection{Ground Control}

The inclusion of 3D ground control points to the BA provides the Geodetic Datum, which ensures that the adjusted DTM has the correct absolute position and scale to match the superior reference frame, here the lunar body-fixed ME-frame. Only five (man-made) features on the nearside of the Moon, i.e. the Apollo and Lunokhod retroreflectors, can be considered control points per definition as they can be identified in the images, and their coordinates are known with cm-accuracy (Williams et al., 2008). Since the LRO mission, the LOLA data have been used as lunar reference on a global scale (Smith et al., 2017). The laser ranging precision of LOLA is $\sim 10 \mathrm{~cm}$. Uncertainties in LRO's position and pointing, however, directly propagate to the positional accuracy of the 3D coordinates of the LOLA shots, and the global accuracy of the LOLA data is approximated to be $<10$ m (Mazarico et al., 2018).

Since laser altimeter shots are not correlated to specific features on the lunar surface and thus are not detectable in the images, their unambiguous use as geodetic reference is limited and can only be achieved indirectly by making "detours" via the object space. Nevertheless, to provide the best available Geodetic Datum to our BA, we use a co-registration technique developed by Gläser et al. (2013). The co-registration plays an important role, as it provides a means to accurately tie LOLA ground points to the image space. For this, the aforementioned initial, SPICE based NAC DTM is generated, and its most probable (mean) position in the geometric center of all intersecting LOLA tracks is determined. This is a multi-step procedure, preceded by an improvement of the relative accuracy of the
LOLA tracks. For further details, the reader is referred to Gläser et al. (2013).

\begin{tabular}{|c|c|c|c|}
\hline LOLA track & $\sigma_{\mathrm{X}}$ in $[\mathrm{m}]$ & $\sigma_{\mathrm{Y}}$ in $[\mathrm{m}]$ & $\sigma_{\mathrm{Z}}$ in $[\mathrm{m}]$ \\
\hline 1371 & 0.6 & 2.1 & 2.3 \\
\hline 2414 & 3.0 & 8.7 & 6.9 \\
\hline 2588 & 0.1 & 8.9 & 10.2 \\
\hline 3457 & 3.4 & 2.2 & 1.6 \\
\hline 3631 & 0.4 & 5.0 & 10.1 \\
\hline 4500 & 1.9 & 5.3 & 1.4 \\
\hline 4848 & 2.9 & 0.1 & 4.3 \\
\hline 5022 & 0.9 & 4.3 & 9.3 \\
\hline 5717 & 2.1 & 2.6 & 0.8 \\
\hline 5891 & 2.7 & 0.2 & 0.2 \\
\hline 6586 & 3.0 & 1.9 & 0.3 \\
\hline 6760 & 0.1 & 0.0 & 0.5 \\
\hline 6934 & 0.1 & 7.9 & 8.9 \\
\hline 7629 & 2.0 & 1.8 & 1.5 \\
\hline 7803 & 4.1 & 2.0 & 3.1 \\
\hline 8498 & 4.2 & 6.7 & 3.5 \\
\hline 9193 & 2.4 & 2.9 & 1.7 \\
\hline 9714 & 0.0 & 6.1 & 5.9 \\
\hline 10235 & 3.4 & 6.3 & 5.5 \\
\hline 10409 & 0.2 & 2.6 & 3.1 \\
\hline 10930 & 0.6 & 10.3 & 13.8 \\
\hline 11105 & 1.6 & 2.6 & 4.2 \\
\hline 11279 & 0.1 & 0.2 & 1.0 \\
\hline
\end{tabular}

Table 2: Standard deviations (in X,Y,Z) of the nominal LOLA tracks covering the Mons Esam region.

After the optimal absolute position of the NAC DTM has been determined, the nominal (measured) LOLA profiles are shifted to accurately match the NAC DTM. The shifted LOLA shots are then assigned to the appropriate photogrammetric object points derived by ray intersection and are thus simultaneously correlated with the corresponding tie points in image space. At the same time, the information about LOLA's nominal, measured $3 \mathrm{D}$ coordinates are preserved to later use them as control points. They are introduced to the BA as unknowns and, in addition, as fictitious observations with individual weights. The necessary a priori standard deviations $\left(\sigma_{\mathrm{X}}, \sigma_{\mathrm{Y}}, \sigma_{\mathrm{Z}}\right)$ are estimated for each original, nominal LOLA track by a variance component estimation within a pre-adjustment.

\subsection{A Priory Accuracies}

Only if the functional model of a least-squares adjustment is complete, the observations are free of gross errors, and only if the stochastic model is correct, statements about the achieved accuracy of the adjustment results are meaningful. Under the assumption that our functional model is correct, we meet the second requirement by repeating the whole procedure until miss-matches of the image matcher are nearly impossible (due to smallest search areas during image matching), and, in addition, by applying an outlier detection method according to Baarda’s Data Snooping (Baarda, 1968). 
The stochastic model, i.e. the a priori knowledge of the accuracy of the individual observations and thus their individual influence (weight) on the result, is known to be a crucial factor in the evaluation of the entire least-squares system. In extraterrestrial photogrammetry these quantities are usually difficult to determine, and the values provided are often associated with an inherent uncertainty. This often leads to incorrect stochastic models resulting in adjustment results that cannot be considered reliable, especially if the empirical variance of unit weight $\mathrm{s}_{0}{ }^{2}$ is not equal to the a priori variance $\sigma_{0}{ }^{2}$. A key component of our BA is the variance component estimation, which after its inclusion has significantly improved the modeling of the a priori standard deviations and thus the reliability and accuracy of our results.

4.6.1 Variance Component Estimation: In our BA we are dealing with different types of observations and different physical units, orders of magnitude, and precision, e.g. with observed image coordinates at $\mu \mathrm{m}$ scale, projection centers at $\mathrm{km}$ scale, rotation angles in radians, and observed LOLA ground coordinates at km scale. All of them have been observed with different precision, and their accuracies must be approximated before the adjustment to be included in the BA in the form of a weight matrix. Following the statistical method called variance component estimation (e.g. Ebner, 1972; Grimm-Pitzinger et al., 1986), we divided the observations into observation groups and assigned individual variances of unit weights to each group.

For example, we assigned a group variance to the tie point coordinates (the biggest group of observations), and, concerning the fictitious observations, we established groups for the coordinates of the NAC-L projection centers (Lagrange nodes), their rotation angles (Lagrange nodes), the NAC-R projection centers, their rotation angles, and, most important, each LOLA track is handled individually and has its own variance of unit weight. During each iteration of the adjustment, the influence of each observation group on the result is distributed according to stochastic aspects, so that after the iterative BA all of the group variances equal $1, \sigma_{0}=\sigma_{\text {tiepoints }}=\sigma_{\text {coord. }}=\sigma_{\text {angles }}=\sigma_{\text {LOLA_track_n }}=1$.

\subsection{Inversion of the Normal Equation Matrix $\mathbf{N}$}

A least-squares adjustment involves the inversion of the normal equation matrix $\mathbf{N}$. Due to the large data sets acquired in extraterrestrial photogrammetry, these matrices are usually very large, and elaborate inversion approaches and tools must be applied. While in the example presented here its size of $4,326 \times 4,326$ is still comparatively small, the computational effort for processing a larger number of adjacent images (an "image block") increases significantly. Therefore, for matrix inversion we use a tool developed at our institute (Burmeister, 2017) that comprises sparse matrix and splitting techniques, Cholesky decomposition, and multi-core parallel computation. By this, the runtime and computational cost could be drastically decreased.

\subsection{General Approach}

The entire procedure of our approach, from image matching to DTM and orthoimage generation, can be applied iteratively. We usually repeat the whole procedure twice, finalized with a LOLA co-registration to evaluate our results.

In the first iteration, the main focus lies on improving the relative orientation of the stereo models in order to obtain improved, coinciding orthoimages for a refined image matching. This will drastically decrease the number of miss-matches, or even eliminate them completely, in the next iteration. In addition, a possible tilt or rotation of the initial DTM will be removed, which could have had a negative effect on the coregistration and thus the $3 \mathrm{D}$ control point assignment. With improved tie point and LOLA control data a second BA is computed.

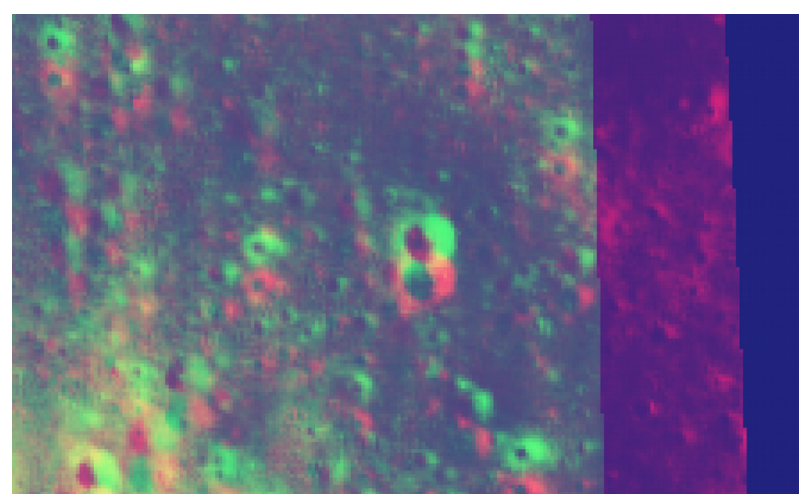

(a)

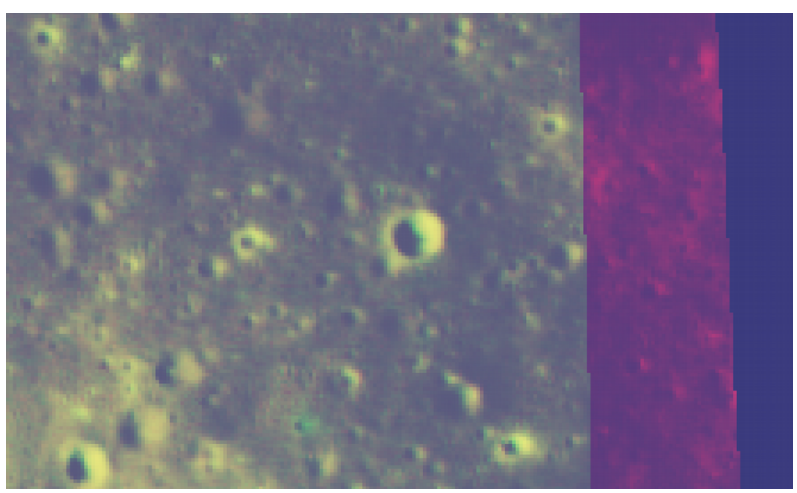

(b)

Figure 3: Image overlay of both NAC-L images acquired from different orbits (map projected and colored); red: M1159863525L; green: M1159877765L. (a) Before adjustment: Errors in the exterior orientation result in y-parallax of about eleven pixels and ambiguous feature locations. (b) After adjustment: The images coincide, there is no visible parallax and accurate feature positions can be derived.

\section{BUNDLE ADJUSTMENT EXAMPLE}

We used the LROC NAC stereo image set M1159863525 and M1159877765 to generate a $3 \mathrm{~m}$ gridded DTM. The images have a pixel scale of $1.2 \mathrm{~m}$ and were acquired from orbits 22860 and 22862 during the Extended Science Mission (ESM) phase. They cover the small mountain named "Mons Esam" located southeast of the Apollo 17 landing site at $14.6^{\circ} \mathrm{N}$ and $35.7^{\circ} \mathrm{E}$.

The initially generated DTM using the most recent kernels provided by NAIF SPICE, the LOLA and LROC teams shows mean ray intersection errors of up to $9.4 \mathrm{~m}$ (Table 1). Figure 2a shows a color-coded view of the intersection errors for each single DTM point before the adjustment. It is obvious that there are serious errors in the exterior orientation, which have a great effect on the derived $3 \mathrm{D}$ points.

23 LOLA tracks passing through the NAC stereo models were co-registered to the initial, SPICE based NAC DTM, and their 3D ground coordinates were automatically related to the appropriate image tie points. Figure 1 shows the geo-locations of the 362 (co-registered) LOLA shots (in black) which were selected to be included in the BA as 3D ground control. In 
white, the same figure shows the distribution of the 1,060 image tie points. 460 of them are two-image-matches evenly covering the area, while 600 triplet matches where added at the NAC-L/ $-\mathrm{R}$ "seams" to stabilize the geometry of the model.

Table 2 lists the a priori standard deviations of the LOLA tracks used in the BA from a variance component estimation in a preadjustment. Overall, these values are in good agreement with the global accuracy of $<10 \mathrm{~m}$ provided by the LOLA team. Due to our combined analysis of the LOLA tracks and the NAC DTM in the BA, we are able to provide (locally) refined and individual standard deviations of the corresponding LOLA tracks.

\begin{tabular}{|l|l|}
\hline observation & a priori standard deviation \\
\hline image tie points $(\mathrm{X}, \mathrm{y})$ & $\sigma_{\mathrm{X}, \mathrm{Y}}=2.5 \mu \mathrm{m}$ \\
\hline NAC-L $(\mathrm{X}, \mathrm{Y}, \mathrm{Z})$ nodes & $\sigma_{\mathrm{X}, \mathrm{Y}, \mathrm{Z}}=66 \mathrm{~m}$ \\
\hline NAC-L $(\omega, \varphi, \mathrm{K})$ nodes & $\sigma_{\omega, \varphi, \mathrm{K}}=0.0258 \mathrm{deg}$ \\
\hline NAC-R $(\mathrm{X}, \mathrm{Y}, \mathrm{Z})$ offset & $\sigma_{\mathrm{X}, \mathrm{Y}, \mathrm{Z}}=1.0 \cdot 10^{-11} \mathrm{~m}$ \\
\hline NAC-R $(\omega, \varphi, \mathrm{K})$ rotation & $\sigma_{\omega, \varphi, \mathrm{K}}=0.0138 \mathrm{deg}$ \\
\hline
\end{tabular}

Table 3: A priori standard deviations.

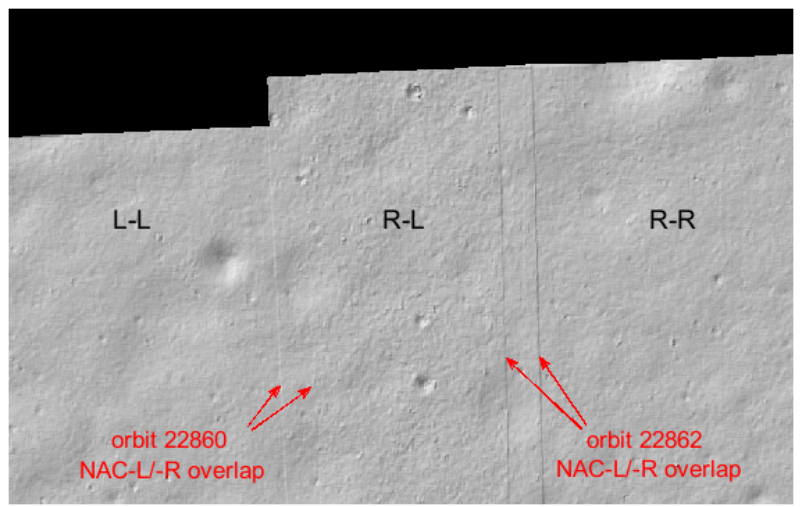

(a)

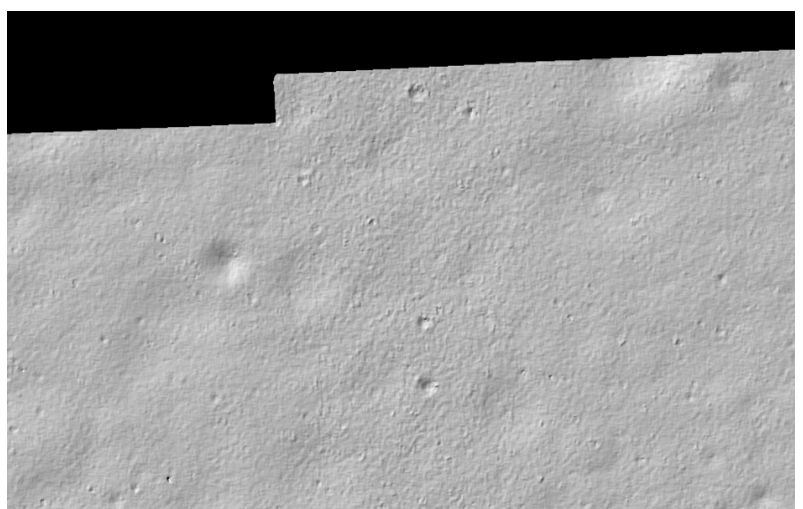

(b)

Figure 4: (a) Upper, central part of the NAC DTM (shaded) before adjustment. Errors in the exterior orientation result in height offsets of up to $\sim 2.6 \mathrm{~m}$ at the edges of the stereo models. (b) After adjustment: Offsets at the model edges are minimized, and the models smoothly merge into each other.

A first BA was performed and the improved exterior orientations were used to process an improved $3 \mathrm{~m} \mathrm{DTM}$ and to rectify orthoimages $(1.2 \mathrm{~m} /$ pixel). When overlaying both NAC$\mathrm{L}$ orthoimages on top of each other, as is shown in Figure 3 before (a) and after the adjustment (b), the improvement of the first BA iteration is clearly visible. While the original orthoimages show high y-parallax of up to eleven image lines, the adjusted orthoimages match nicely, i.e. the image rays intersect within pixel scale. Using the improved orthoimages we ran the image matcher again, allowing only smallest search areas of 1 pixel. This way, we highly decrease the chance of miss-matches.

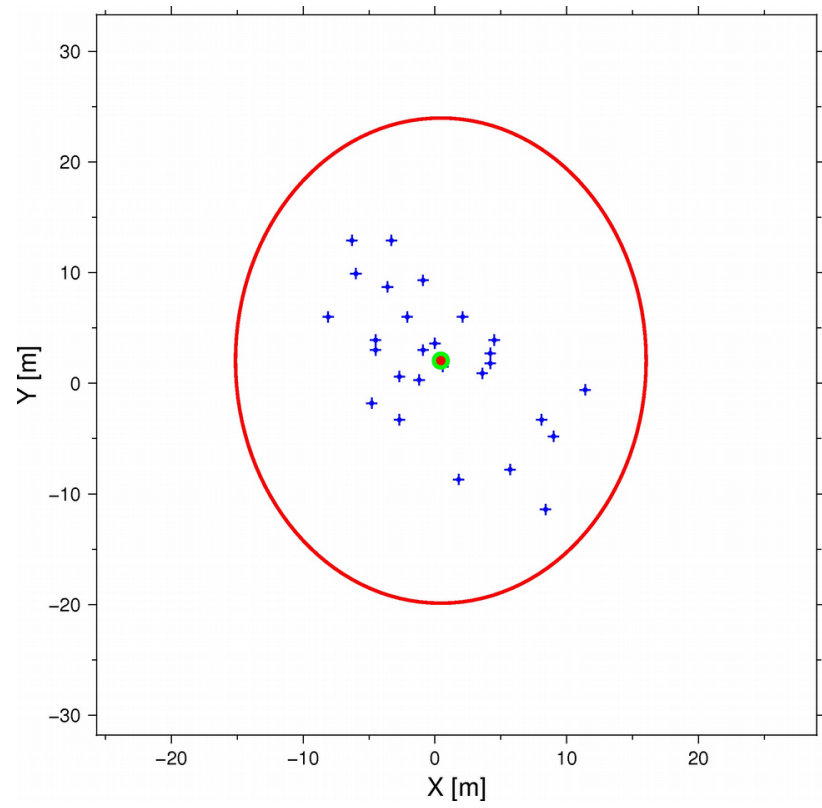

Figure 5: The individual 2D displacement of each LOLA track required in order to match with the NAC DTM (blue plus signs). The final position of the adjusted NAC DTM (green dot) lies in the geometric center (red dot) of all available LOLA tracks whose shift values lie within the $3 \sigma$ range (red ellipse). The remaining shift values are $0.4 \mathrm{~m}$ in X-and $2.0 \mathrm{~m}$ in Y-direction, and are well within one DTM pixel (3 m).

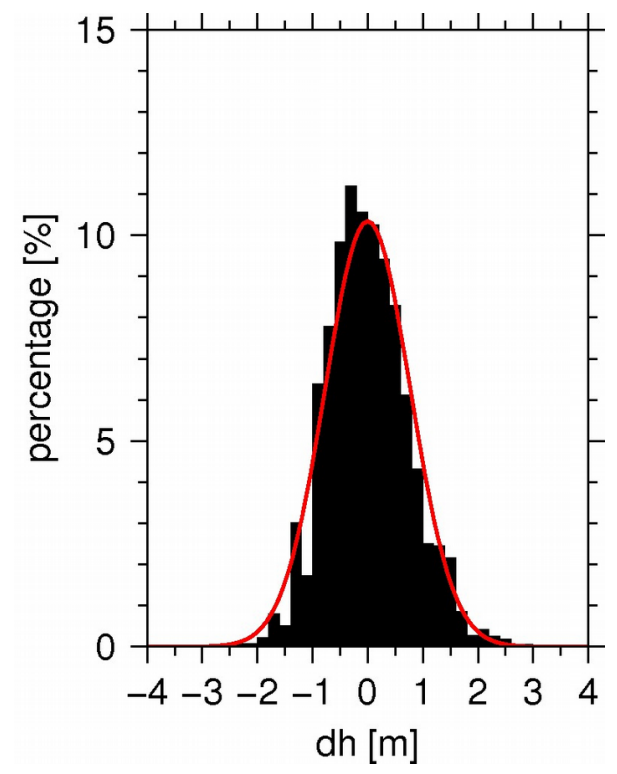

Figure 6: The height residuals of all available (registered) LOLA shots and the adjusted LROC NAC DTM are normal distributed around a mean of $\sim 0.0 \mathrm{~m}$ with a standard deviation $(1 \sigma)$ of $0.77 \mathrm{~m}$. 


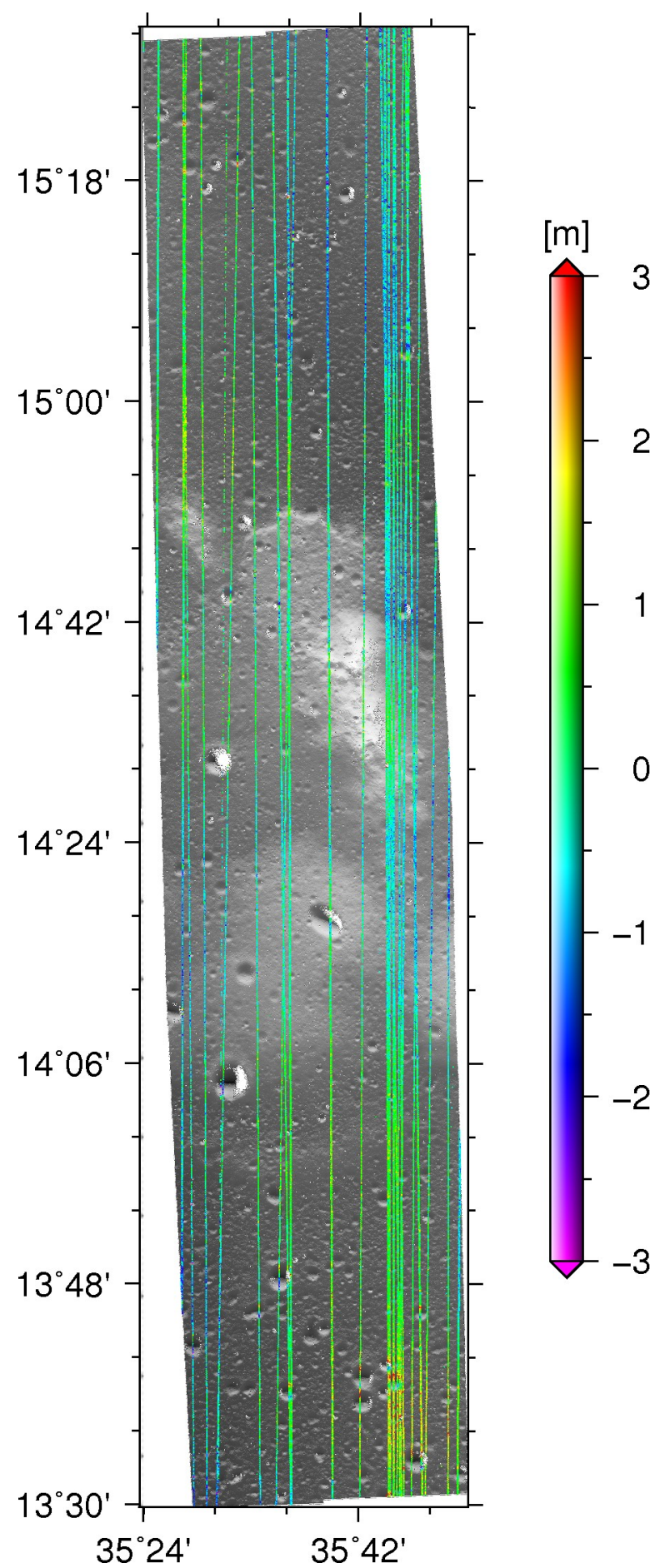

Figure 7: LOLA tracks are color-coded by the height differences of the LOLA shots with the underlying, adjusted LROC NAC DTM. The DTM agrees very well with the registered LOLA tracks, and the majority of the points agree within $\pm 1 \mathrm{~m}$.

We also used the adjusted DTM to perform a second coregistration, since the improved relative and absolute accuracy of the adjusted DTM will result in higher registration precision. The new tie points as well as newly co-registered LOLA points serve as input data for a second, final BA. Their weights are assigned according to the pre-adjustment and are given in Tables 2 and 3.

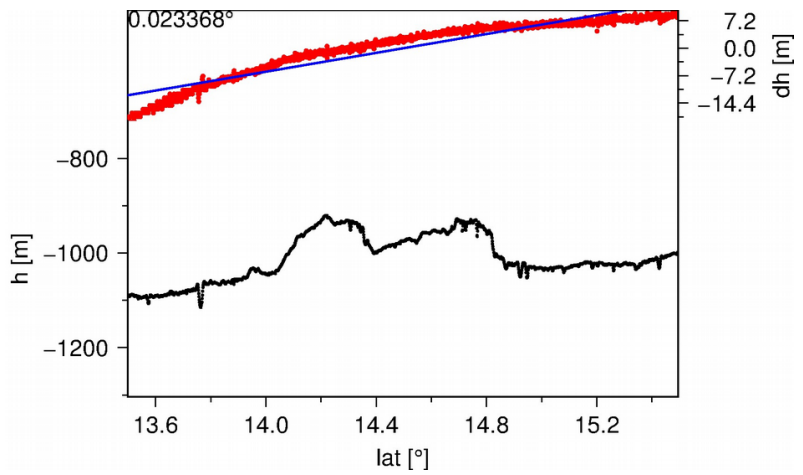

(a)

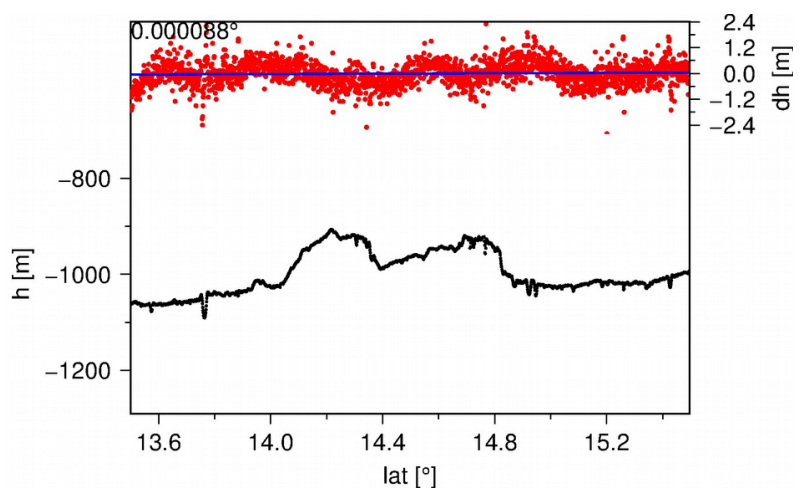

(b)

Figure 8: Comparison of height residuals (red) of LOLA shots of track \#8498 with the adjusted LROC NAC DTM before (a) and after adjustment (b). The corresponding profile (black) is given to provide an overview of the local topography. An initial tilt of the NAC DTM of $\sim 0.023^{\circ}$ (a) in along track direction (approximated by the blue line) was eliminated by the BA (b). Elevation is given relative to the mean lunar radius of $1737.4 \mathrm{~km}$.

Subsequent to the final BA, an adjusted LROC NAC DTM $(3 \mathrm{~m})$ and the corresponding ortho images $(1.2 \mathrm{~m} / \mathrm{pixel})$ are processed using the improved exterior orientations. The mean 3D point error of the object points was estimated by the BA to be $0.95 \mathrm{~m}$ (RMS), and the forward ray intersections show highly improved intersection accuracies of $0.14 \mathrm{~m}$, on average (Table 2). In Figure 2b, the improved exterior orientations for each of the 52,224 image lines of all four images nearly result in the optimum of a homogeneously black representation.

Furthermore, and this is probably the most obvious positive effect that the BA has on the NAC DTM, the "seams" at the center model edges, where the four NAC stereo models overlap, are eliminated in the adjusted DTM. This is where errors in the geometric model are most likely to show, and the SPICE based DTMs usually show significant height differences. In the example presented, the average height offsets ranged from $\sim 1.0 \mathrm{~m}$ to $\sim 2.6 \mathrm{~m}\left(\sigma_{\text {offset }}=0.4 \mathrm{~m}\right)$. In Figure $4 \mathrm{a}$ these elevation edges are clearly visible in the initial DTM and are almost completely eliminated $\left(<0.1 \mathrm{~m}, \sigma_{\text {offset }}=0.4 \mathrm{~m}\right)$ after we applied our iterative BA approach (Figure $4 \mathrm{~b}$ ). It should be noted that we do not use filters or the like to smooth the data after DTM interpolation. 


\subsection{Absolute Accuracy of the Adjusted DTM}

To check and verify the absolute accuracy that we achieved by applying our approach, a final co-registration with all available LOLA tracks is performed.

By using the nominal (measured) 3D coordinates of the LOLA shots as ground control, we achieve the statistically most probable position and orientation of the NAC DTM relative to the selected LOLA tracks. This is impressively shown in Figure 5 where we report the 2D shift of each LOLA track to match the NAC DTM. Here, the BA accurately placed the DTM in the middle of the geometric center of the LOLA tracks. The remaining $2 \mathrm{D}$ offset is $0.4 \mathrm{~m}$ in $\mathrm{X}-, 2.0 \mathrm{~m}$ in Y-direction, and $0.25 \mathrm{~m}$ in height (not shown in Figure 5).

In addition, we calculated the height differences of all (registered) LOLA shots with the adjusted NAC DTM. In the example presented here, the height differences are normal distributed around a mean value of $\sim 0.0 \mathrm{~m}$ with a standard deviation $(1 \sigma)$ of $0.77 \mathrm{~m}$ (Figure 6 ). This overall excellent agreement is also shown in Figure 7, where the height residuals are color-coded and plotted on top of the shaded DTM.

For further analysis we determined the height residuals of each LOLA track with the adjusted DTM. Figure 8, for example, shows the residuals of LOLA track \#8498 with an initial tilt of $\sim 0.023^{\circ}$ before adjustment (Figure $8 \mathrm{a}$ ), which was completely eliminated by our BA (Figure 8b). Nevertheless, small errors remain which could not yet be addressed by our approach. Possible sources are low frequency oscillation of the solar panels (jitter; Mattson et al., 2011) which will be investigated in future studies.

\section{SUMMARY AND CONCLUSION}

The TU Berlin group of the LROC team successfully implemented a least-squares BA for spaceborne CCD line-scan camera systems that rigorously models the geometric properties at the moment of image acquisition. The BA was applied to LROC NAC images, and the example presented shows that the overall geometry of the stereo models could be significantly improved.

Mean intersection accuracies of initially up to $9.4 \mathrm{~m}$ were homogenized for the incorporated stereo image pair and improved to $0.14 \mathrm{~m}$ on average (just $\sim 5 \%$ of the pixel size in the presented example). The BA estimated the mean point error of the adjusted 3D object points to be $0.95 \mathrm{~m}$, and the mean height offsets at the narrow NAC-L/-R image overlap of initially up to $2.6 \mathrm{~m}$ were reduced to less than $0.1 \mathrm{~m}$.

The inclusion of $\sim 360$ LOLA shots from 23 tracks as ground control, accurately tied to image space by an initial coregistration, allowed us to register the final adjusted NAC DTM to the currently most accurate global reference frame. The resulting standard deviation of the normal distributed height residuals was $0.77 \mathrm{~m}$. The BA also provides accuracy assessments for each nominal LOLA track, which were used for registration during the adjustment. This will also be useful for the evaluation of further LOLA derived products.

\section{ACKNOWLEDGEMENTS}

We gratefully acknowledge the LRO mission operation team, the LROC science team at Arizona State University, and the LOLA science team. Isabel Haase was funded by the Federal
Ministry for Economic Affairs and Energy (FKZ 50OW1702) and Philipp Gläser by a grant of the German Research Foundation (GL 865/2-1).

\section{REFERENCES}

Baarda, W., 1968. A testing procedure for use in geodetic networks. Neth. Geod., Comm., Publ. Geodesy, New Series, 2, pp. 1-97.

Burmeister, S., 2017. Determining rotational elements of planetary bodies. Technische Universität Berlin, Dissertation, pp. 1-121.

Ebner, H., 1972. A posteriori Varianzschätzungen für die Koordinaten unabhängiger Modelle. Zeitschrift für Vermessungswesen, 97, pp. 166-172.

Ebner, H., et al., 1992. A Simulation Study on Point Determination for the MOMS-02/D2 Space Project Using an Extended Functional Model. ISPRS Archives - Volume XXIX Part B4, pp. 458-464.

Gläser, P., et al., 2013. Co-registration of laser altimeter tracks with digital terrain models and applications in planetary science. Planetary and Space Science, 89, pp. 111-117.

Grimm-Pitzinger, A. et al., 1986. Bedeutung der Varianzkomponentenschätzung für die geodätische Praxis, Österreichische Zeitschrift für Vermessungswesen und Photogrammetrie, 74, pp. 101-112.

Haase, I., et al., 2019. Coordinates and Maps of the Apollo 17 Landing Site. Earth and Space Science, 6(1), pp. 59-95.

Keller, J. W., et al., 2016. The Lunar Reconnaissance Orbiter Mission - Six years of science and exploration at the Moon. Icarus, 273, pp. 2-24.

Mattson, S. S., et al., 2011. Continuing Analysis of Spacecraft Jitter in LROC NAC. 42th LPSC, abstract \#2756.

Mazarico, E., et al., 2018. Orbit determination of the Lunar Reconnaissance Orbiter: Status after seven years. Planetary and Space Science, 162, pp. 2-19.

Robinson, M. S., et al., 2010. Lunar Reconnaissance Orbiter Camera (LROC) Instrument Overview. Space Science Reviews 150, pp. 81-124.

Scholten, F., et al., 2005. Mars express HRSC data processing Methods and operational aspects. PE\&RS, 71(10), pp. 11431152 .

Smith, D. E., et al., 2017. Summary of the results from the lunar orbiter laser altimeter after seven years in lunar orbit. Icarus 283, pp. 70-91.

Speyerer, E. J., et al., 2014. New Spice To Improve The Geodetic Accuracy Of LROC NAC And WAC Images, 45th LPSC, abstract \#2421.

Speyerer, E. J., et al., 2016. Pre-flight and On-orbit Geometric Calibration of the Lunar Reconnaissance Orbiter Camera. Space Science Reviews 200, pp. 357-392.

Williams, J. G., et al., 2008. DE421 Lunar Orbit, Physical Librations, and Surface Coordinates. JPL IOM 335-JW, DB, WF-20080314-001, pp. 1-14. 